\title{
Formulation and evaluation of Antiwrinkle activity of Cream and Nano emulsion of Moringa oleifera seed oil
}

\author{
${ }^{1}$ Duraivel S, ${ }^{2}$ Sk. Asma shaheda ${ }^{2}$, Sk. Rabbani Basha ${ }^{3}$, Sk. Eesaf Pasha ${ }^{3}$, Sk. \\ Jilani $^{3}$ \\ ${ }^{1}$ Principal, Nimra College of Pharmacy, Jupudi, Ibrahimpatnam Vijayawada-521 456, A.P. \\ ${ }^{2}$ Dept. of Pharmaceutics, Nimra College of Pharmacy, Jupudi, Ibrahimpatnam Vijayawada-521 456, A.P. \\ ${ }^{3}$ Dept. of Pharmacology, Nimra College of Pharmacy, Jupudi, Ibrahimpatnam Vijayawada-521 456, A.P.
}

\begin{abstract}
Aging is a natural phenomenon that leads to various changes in the physiology of the skin. The changes occurring in the physiology of the skin makes the candidate to appear old. Application of antiaging creams is the best choice even though various treatment methods are available because it nourishes skin and prevents or repairs fine lines and wrinkles thus giving young looking appearance. Apart from that, nature has an excellent anti-aging remedies that acts externally whereas internally to delay aging signs and some will act to repair and prevent aging signs. In the present study, Moringa oleifera was studied for its antiaging benefits as the seed oil is rich in antioxidants that might prevent the oxidative damage of the skin. By using the Moringa seed oil in various ratios, cream and nano emulsion were prepared and they are characterised for its physical properties. The best formula was optimised which has been evaluated for antiaging activity using animal models by topical application of the formulations for two times a day upto 30days and the results were compared with the standard. The results showed that the nano emulsion formulation was found more efficacious than the cream formulation. This shows that the moringa oil has a good antiaging activity and donot showed any irritant effects on skin.
\end{abstract}

Key Words: Wrinkles, Premature skin aging, Moringa oleifera, Oxidative damage, Skin topography analysis.

\section{Introduction}

Aging can be noticed by seven key signs like fine lines and wrinkles, changes in skin tone and texture, skin surface dullness, visible pores, Blotchiness, age spots and Dryness. Among all these signs, appearance of fine lines and wrinkles on skin is the common and most prominent sign of aging. So skin creams used to prevent aging signs are also called as Anti-wrinkle creams. Skin consists of three layers Epidermis, Dermis and Subcutaneous layer. Dermis of the skin consists of elastin fibres which will maintains the skin structure by stretching and folding back when the muscle undergo various stress conditions like facial expressions etc. Collagen, also present in Dermis is responsible for preventing wrinkle formation. Upon aging, the skin will lose elastin and also Collagen undergoes breakdown ${ }^{1}$. This makes the skin thinner and moisture cannot enter the skin layers making the skin drier. The subcutaneous fat which gives the skin the plumpy appearance also begins to disappear. All these conditions will leads to progression of wrinkle formation which is due to the structural changes in lower dermal layers of the $\operatorname{skin}^{2}$ which is shown in the table $\mathbf{1}$ and figure $\mathbf{1 .}$

Aging is of two types: Chronological aging or Intrinsic aging and Photo aging ${ }^{3}$. Photo-aging also called as premature skin aging ${ }^{4}$ is caused by continuous exposure of the skin to solar UV irradiation. It results in several skin symptoms, such as leathery texture, mottled pigmentation, and wrinkles. Apart from Intrinsic and photoaging, so called stochastic aging ${ }^{5}$ connotes cell damage by metabolic processes, free radicals and cosmic irradiation. Hence, reducing oxidative stress has been the major focus of anti-aging research, and the antioxidant supplements are recommended based on this research. Moreover, these antioxidants are active both topically and internally, which means they can be applied in form of creams, gels, or taken as capsules or tablets. Antioxidants are helpful to achieve efficient free radical scavenging, since they relieve one another of free radical burden. Vitamin E, Vitamin C, lipoic acid, coenzyme Q10, nicotinic acid, and glutathione neutralize free radicals by different methods, and they complement one another's efforts.

Based on this data ${ }^{5}$, Moringa oleifera was choosed for the present study inorder to evaluate the antiwrinkle efficacy of the seed oil which is rich in antioxidants ${ }^{7}$. Using the seed oil of M. Oleifera, cream and nano-emulsion were prepared and studied for antiwrinkle efficacy. As the prepared formulation is a herbal formulation it may reduce the side effects of the chemical based marketed formulations. 


\section{Materials and Methods:}

2.1. Plant Material: The seeds of Moringa oleifera Fam (Moringaceae) were collected from local region of Vijayawada, Andhra Pradesh, India, in june 2013. The plant material was identified and authenticated by Dr. P. G. Diwakar Botanical survey of India, Hyderabad (Ref no. BSI/WC/Tech/2013/370).

Moringa oleifera seeds (Figure 2 ) were collected from surroundings of Vijayawada. Pods and shells were removed manually and kernels were grounded in a domestic blender (Preethi) and sieved through $600 \mu \mathrm{m}$ stainless steel sieve. Solvent selected for extraction of oil was 95\% ethanol (Et).

\subsection{Oil Extraction procedure:}

Manual (man): Moringa oleifera seeds were defatted by using $95 \%$ ethanol in $5 \%(\mathrm{w} / \mathrm{v})$ suspension, mixing with a magnetic stirrer for 60 minutes. Supernatant was separated by centrifugation (3000 rpm, $45 \mathrm{~min}$ ) and the settled powder was dried at room temperature for 24 hours.

Continues (Sx): About $50 \mathrm{~g}$ of M. oleifera crushed seeds were fed to a lab-scale Soxhlet extractor fitted with a 1-L round-bottom flask and a condenser. The extraction was executed for 6 hours with $350 \mathrm{~mL}$ of solvent. The extracted oil yield was expressed as percentage, which is defined as weight of oil extracted over weight of the sample taken.

2.3. Cream formulation: Oil in water $(\mathrm{O} / \mathrm{W})$ emulsion-based cream (semisolid formulation) was formulated. The emulsifier (stearic acid) and other oil soluble components (Cetyl alcohol, liquid paraffin) were dissolved in the oil phase (Part A) and heated to $75^{\circ} \mathrm{C}$. The preservatives and other water soluble components (Methyl paraben, Glycerol, Propylene glycol, ethanol extract of Moringa oleifera seeds were dissolved in the aqueous phase (Part B) and heated to $75^{\circ} \mathrm{C}$. After heating, the aqueous phase was added in portions to the oil phase with continuous stirring until cooling of emulsifier took place. The formula for the cream was given in table 2. The formulated cream was subjected to the following evaluation parameters such as $\mathrm{pH}$ determination, viscosity, Dye test, Homogenity, appearence, after feel, Type of smear, removal, acid value, saponification value, irritancy test and Accelerated stability analysis.

\subsection{Preparation of Moringa oleifera seed oil nano-emulsion (MONE)}

An emulsion was prepared following the formula in the ingredient percentages (Table 3). The following materials were used to produce the emulsion; Water, Moringa oleifera seed oil, and Sodium caseinate. Sodium caseinate was used since it is a natural emulsifier (flexible protein) which has been used to produce nano-emulsions. Addition rate $(\% \mathrm{w} / \mathrm{w})$ of the ingredients was modified to emulsify the highest percent of MONE and produce the lowest droplet size (nm) without affecting the emulsion stability. Sodium caseinate was dissolved in water and stirred using a magnetic stirrer at $700 \mathrm{rpm}$ for $1 \mathrm{hr}$. at room temperature. Moringa oleifera seed oil was added gradually into the sodium caseinate solution and mixed by stirring for $5 \mathrm{~min}$. An ultrasonic processor set at $80 \%$ amplitude was used to produce a stable micro-emulsion to process each batch $(100 \mathrm{~mL} / \mathrm{batch})$ of solution in a $250 \mathrm{~mL}$ glass beaker for about $10 \mathrm{~min}$. The emulsion was constantly cooled to maintain a temperature of approximately $20^{\circ} \mathrm{C}$ by means of immersion in an ice bath during the sonication process. The emulsion was then processed by a mechanical stirrer device to produce a nano-emulsion. Emulsion batches $(100 \mathrm{~mL} / \mathrm{batch})$ in a $200 \mathrm{~mL}$ beaker was sheared at $16,000 \mathrm{rpm}$ for $10 \mathrm{~min}$ to reduce droplet size to nano-scale. The beaker was set in an ice bath to maintain a temperature of approximately $20{ }^{\circ} \mathrm{C}$ during mechanical stirring. An emulsion produced only by ultrasonication was prepared as a control. The nanoemulsion and the control emulsion were kept refrigerated at $5{ }^{\circ} \mathrm{C}^{7}$. Composition Moringa oleifera Nanoemulsion Formulations was given in the Table 3. It was characterized for the following parameters such as particle size, Particle surface charge (zeta potential), transmission electron microscopic studies (TEM).

2.5. Evaluation: By performing the above mentioned evaluation tests, the best formula was optimised, and it was subjected to further evaluation of antiwrinkle activity by using animal models. Various tests that can be performed to evaluate the antiwinkle efficacy are: Wrinkle score measurement, Histological evaluation, Invitro trial - Hydrating and Anti-Wrinkle efficacy, Staining, Anti-wrinkle test on healthy mice using silicon imprints, Instrumental assessment using tewameter MPA 5, Skin moisture and TEWL, Surface Evaluation of Living Skin (SELS), volume and energy which can be studied using Visioscan ${ }^{\circledR}$ VC 98/ software SELS 2000.

In the present study the following method was used for evaluating the antiwrinkle efficacy of the prepared formulation:

Study of Anti-wrinkle efficacy using animal models by analysis of Photographs and by Staining method: (Test was performed at Albino research and training institute, Hyderabad, Andhra pradesh, India) Fourweek-old female hairless mice (SKH-1) weighing 17-24 g were obtained from Indian Institute of Toxicology Research Lucknow. Mice had free access to food and water and were acclimated to the air-conditioned room (23 $\pm 2^{\circ} \mathrm{C}$ and $50 \pm 10 \%$ humidity with a $12 \mathrm{~h}$ light $/ 12 \mathrm{~h}$ dark cycle) for 1 week before the in vivo anti-wrinkle study. 
Firstly, the animals under study are subjected to UVB irradiation at suberythemal doses for upto 2 weeks at $345 \mathrm{~nm}$ which will produce marked wrinkling ${ }^{8,9,10}$. Skin topography analysis was performed on the skin surface of mice before and after treatment for measuring the effectiveness of $M$. Olifera. The same skin areas were examined before the process (Day 0) and after the process (Dayl, Day7, Day15 and Day 30), by taking photographs and also by means of observation under magnifying glass (Leica M LZIII, augment 1OX) with outer white light.

The mouse was divided into two groups, those with male mouse and those with female mouse, $50 \%$ of the mouse in the study are male and the other $50 \%$ female. The test was performed under the environmental conditions between $23-25^{\circ} \mathrm{C}$ and relative humidity of $60 \%$.

Two types of trials were carried out:

1. It was studied whether the two formulations could affect the aqueous content of the skin and

2. The evaluation of the wrinkle, in the short-term and long-term study.

Short-term trial: the formulas were applied for every 12 hours and measurements were taken at the end of 24 hours after application.

Long-term trial: To do this, the subjects were treated with the cream for one month, twice a day. The evaluation of the aqueous content of the skin was determined after 24 hours , 7, 15, 21 and 30 days.

In the present study only the wrinkle evaluation was done in long-term study. The appearance of the skin depends on its general condition, and in particular its level of moisturisation, as this regulates the elasticity, flexibility and smoothness of the skin. The integrity of the extracellular matrix, especially the collagen fibres, is essential to ensure that the epidermis is firmly anchored to the dermis. It has been suggested that the deterioration of the collagen fibres, at the dermis-epidermis union, weakens it, which finally leads to the appearance of wrinkles. In expression wrinkles, the contraction of the muscle leads to contraction of the fibroblasts and with this the contraction of the collagen fibres occurs and even deterioration of the extracellular matrix also occurs in the affected area. Consequently, the hydrating properties of all the components of the matrix will be affected.

Antiwrinkle creams should be capable of relaxing the fibroblasts, which will decontract the collagen and elastin matrix, which could be decisive in preventing deterioration of the functions of collagen, such as water retention. Thus, it could be deduced that by reducing the wrinkle, greater moisturisation is achieved.

Photographs of a wrinkle of mouse with male and female, respectively, are taken in the beginning and upto 30 days of treatment. In the photographs, it is possible to observe that the formulations under study improved the appearance of the wrinkles.

After 30 days treatment with the topical preparations, the photographs were observed (Figure-8 and figure-11). There was no skin changes in Group-1 animals. In Group-2 (negative control, saline with UV irradiation) thick and deep wrinkles were observed. In contrast, Group-3 (positive control, RESIST Intensive Wrinkle-Repair Retinol Serum with UV irradiation) showed better anti-wrinkle scores. The Group-4 (MONE 3 with UV irradiation) showed significantly better anti-wrinkle scores compared with standard anti wrinkle cream. The Group-5 (Formulation 3 Moringa oleifera Oil based cream with UV irradiation) showed smooth and improved skin surfaces. Results after the 30day study of all the groups was shown in the

III. Results and Discussions:

The result data was tabulated in Table No: 4- 12 and shown in Figure No: 3-11.

Table 1: Skin structure and their observed effects of aging ${ }^{1}$

\begin{tabular}{|c|c|}
\hline Skin structure & Observed effects of aging \\
\hline & Lower lipid content was observed \\
\hline Epidermis & $\begin{array}{l}\text { 1. Dermal-epidermal junction flattens } \\
\text { 2. Number of enzymatically active melanocytes decreases by } 8 \% \text { to } 20 \% \text { per } \\
\text { decade } \\
\text { 3. Number of langerhans cells decreases } \\
\text { 4. Capacity for re-epithelization diminishes } \\
\text { 5. Number of pores increases }\end{array}$ \\
\hline Dermis & $\begin{array}{l}\text { 1. Thickness reduced (atrophy) } \\
\text { 2. Vascularity and cellularity decrease } \\
\text { 3. Collagen synthesis decreases } \\
\text { 4. Pacinian and meissner's corpuscles degenerate } \\
\text { 5. Structure of sweat glands becomes distorted, number of functional sweat } \\
\text { glands of decreases }\end{array}$ \\
\hline
\end{tabular}


Formulation and evaluation of Antiwrinkle activity of Cream and Nano emulsion of Moringa oleifera

\begin{tabular}{|c|c|}
\hline & $\begin{array}{l}\text { 6. Elastic fibers degrade } \\
\text { 7. Number of blood vessels decreases and number of nerve endings reduced }\end{array}$ \\
\hline Hypodermis & $\begin{array}{l}\text { Distribution of subcutaneous fat changes and overall volume of fat } \\
\text { decreases. }\end{array}$ \\
\hline Appendages & $\begin{array}{l}\text { 1. Number of sweat glands decreases } \\
\text { 2. Nail plates become abnormal } \\
\text { 3. Sebum production reduced } \\
\text { 4. } \\
\text { Hair loses normal pigments and thins up }\end{array}$ \\
\hline
\end{tabular}

Table 2: Composition Moringa oleifera oil based cream

\begin{tabular}{|c|c|c|c|c|c|c|}
\hline \multirow[t]{2}{*}{ S.No } & \multirow[b]{2}{*}{ Ingredients } & \multicolumn{5}{|c|}{ Formula \% w/w } \\
\hline & & F1 & F2 & F3 & F4 & F5 \\
\hline 1. & $\begin{array}{l}\text { Extracted seed oil of } \\
\text { Moringa oleifera }\end{array}$ & 2 & 2 & 2 & 2 & 2 \\
\hline 2. & Stearic acid & 10 & 8 & 6 & 4 & 2 \\
\hline 3. & Cetyl alcohol & 6 & 6 & 6 & 6 & 6 \\
\hline 4. & Liquid paraffin & 6.6 & 6.6 & 6.6 & 6.6 & 6.6 \\
\hline 5. & Glycerol & 3 & 3 & 3 & 3 & 3 \\
\hline 6. & Methyl paraban & 0.02 & 0.02 & 0.02 & 0.02 & 0.02 \\
\hline 7. & Propylene glycol & 30 & 30 & 30 & 30 & 30 \\
\hline 8. & Water, qs, 100 & Qs & qs & qs & qs & qs \\
\hline
\end{tabular}

Table 3: Composition Moringa oleifera Nano-emulsion Formulations

\begin{tabular}{|c|c|c|c|c|c|c|}
\hline \multirow{2}{*}{ S.No } & \multirow{5}{|c|}{ Ingredients } & \multicolumn{5}{|c|}{ Amount (\%)* } \\
\cline { 3 - 7 } & & MONE 1 & MONE 2 & MONE 3 & MONE 4 & MONE 5 \\
\hline 1. & Moringa oleifera oil & 5 & 10 & 15 & 20 & 25 \\
\hline 2. & Sodium caseinate & 10 & 10 & 10 & 10 & 10 \\
\hline 3. & Water & 85 & 80 & 75 & 70 & 65 \\
\hline 4. & Total & 100 & 100 & 100 & 100 & 100 \\
\hline
\end{tabular}

Table 4: Oil yield extraction for both methods and solvents

\begin{tabular}{|c|c|c|}
\hline S.No & Method & \% of Seed Oil yield extraction (w/w) \\
\hline 1. & Manual extraction & 17.81 \\
\hline 2. & Soxhlet extraction & 32.11 \\
\hline
\end{tabular}

Table 5: a:Physical parameter of $F 1$ cream on room and accelerated temperature

\begin{tabular}{|c|c|c|c|c|c|c|c|c|c|}
\hline $\begin{array}{c}\text { Formulatio } \\
\mathbf{n}\end{array}$ & Days & $\begin{array}{c}\text { Temperatur } \\
\text { e }\end{array}$ & pH & $\begin{array}{c}\text { Homogeni } \\
\text { ty }\end{array}$ & Appearance & $\begin{array}{c}\text { Spreadibilit } \\
\mathbf{y}\end{array}$ & After feel & $\begin{array}{c}\text { Type } \\
\text { of } \\
\text { smear }\end{array}$ & Removal \\
\hline \multirow{8}{*}{$\mathrm{F} 1$} & \multirow[t]{2}{*}{0} & RT & 5.7 & Good & $\begin{array}{l}\text { No change } \\
\text { in colour }\end{array}$ & Good & Emollient & $\begin{array}{c}\text { Non } \\
\text { greasy }\end{array}$ & Easy \\
\hline & & $40^{\circ} \mathrm{C} \pm 1{ }^{\circ} \mathrm{C}$ & 5.6 & Good & $\begin{array}{l}\text { No change } \\
\text { in colour }\end{array}$ & Good & Emollient & $\begin{array}{c}\text { Non } \\
\text { greasy }\end{array}$ & Easy \\
\hline & \multirow[t]{2}{*}{5} & RT & 5.7 & Good & $\begin{array}{l}\text { No change } \\
\text { in colour }\end{array}$ & Good & Emollient & $\begin{array}{l}\text { Non } \\
\text { greasy }\end{array}$ & Easy \\
\hline & & $40^{\circ} \mathrm{C} \pm 1{ }^{\circ} \mathrm{C}$ & 5.6 & Good & $\begin{array}{l}\text { No change } \\
\text { in colour }\end{array}$ & Good & Emollient & $\begin{array}{c}\text { Non } \\
\text { greasy }\end{array}$ & Easy \\
\hline & \multirow[t]{2}{*}{10} & RT & 5.8 & Good & $\begin{array}{l}\text { No change } \\
\text { in colour }\end{array}$ & Good & Emollient & $\begin{array}{l}\text { Non } \\
\text { greasy }\end{array}$ & Easy \\
\hline & & $40^{\circ} \mathrm{C} \pm 1{ }^{\circ} \mathrm{C}$ & 5.7 & $\begin{array}{c}\text { Satisfactor } \\
y\end{array}$ & $\begin{array}{l}\text { No change } \\
\text { in colour }\end{array}$ & Good & Emollient & $\begin{array}{l}\text { Non } \\
\text { greasy }\end{array}$ & Easy \\
\hline & \multirow[t]{2}{*}{15} & RT & 5.9 & Good & $\begin{array}{l}\text { No change } \\
\text { in colour }\end{array}$ & Good & Emollient & $\begin{array}{c}\text { Non } \\
\text { greasy }\end{array}$ & Easy \\
\hline & & $40^{\circ} \mathrm{C} \pm 1{ }^{\circ} \mathrm{C}$ & 5.7 & Satisfactor & No change & Good & Emollient & Non & Easy \\
\hline
\end{tabular}


Formulation and evaluation of Antiwrinkle activity of Cream and Nano emulsion of Moringa oleifera

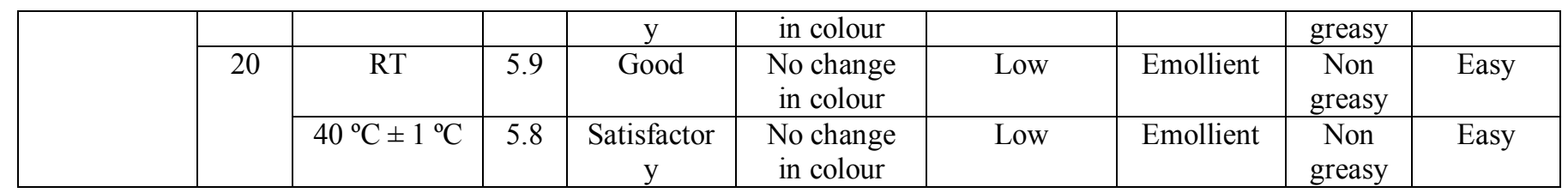

Table 5: b:Physical parameter of F2 cream on room and accelerated temperature

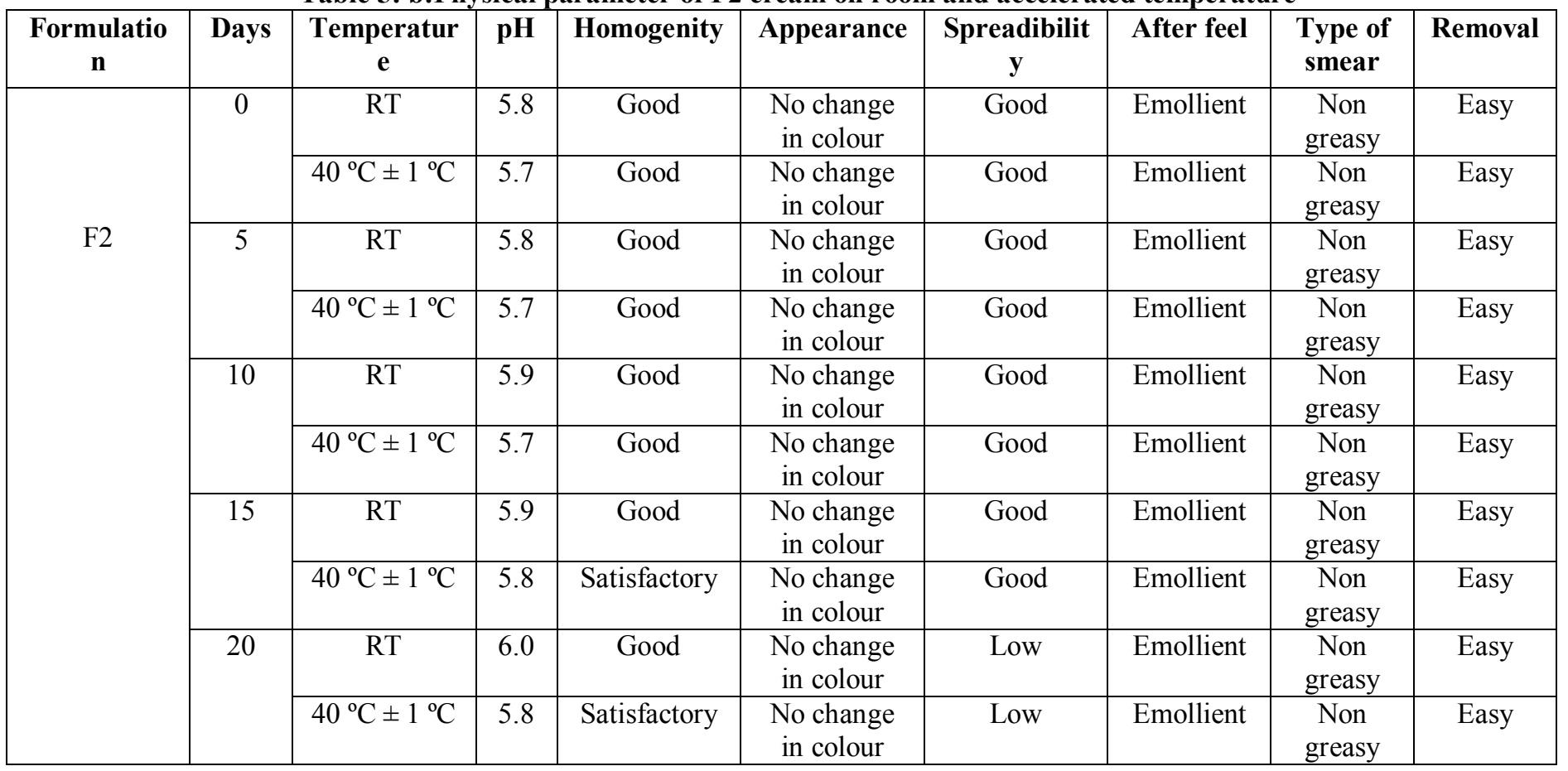

Table 5:c: Physical parameter of F3 cream on room and accelerated temperature

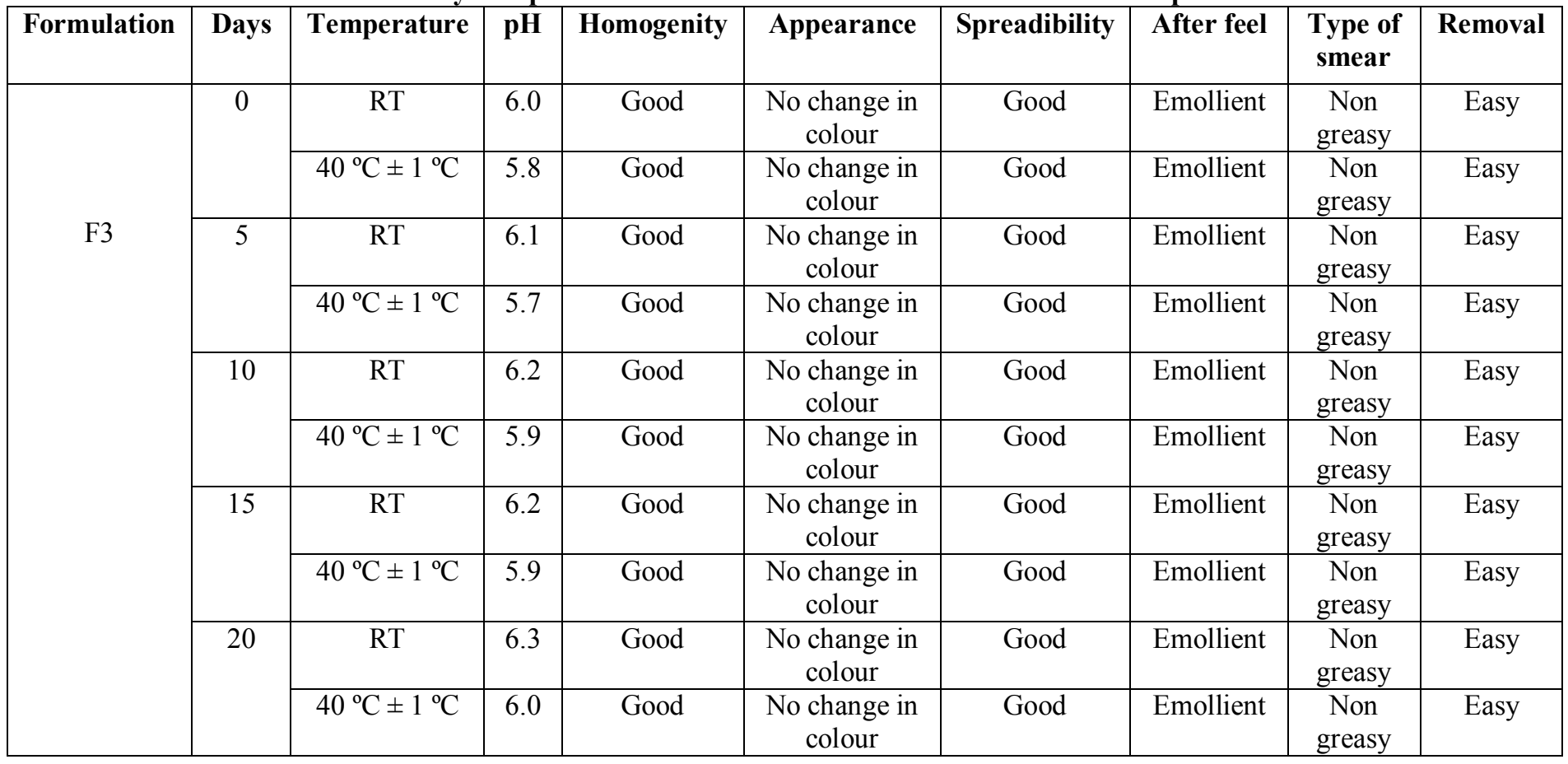


Table 5:d: Physical parameter of F4 cream on room and accelerated temperature

\begin{tabular}{|c|c|c|c|c|c|c|c|c|c|}
\hline Formulation & Days & Temperature & pH & Homogenity & Appearance & Spreadibility & $\begin{array}{c}\text { After } \\
\text { feel }\end{array}$ & $\begin{array}{l}\text { Type } \\
\text { of } \\
\text { smear }\end{array}$ & Removal \\
\hline \multirow{10}{*}{ F4 } & \multirow[t]{2}{*}{0} & RT & 6.6 & Good & $\begin{array}{l}\text { No change } \\
\text { in colour }\end{array}$ & Good & Emollient & $\begin{array}{l}\text { Non } \\
\text { greasy }\end{array}$ & Easy \\
\hline & & $40^{\circ} \mathrm{C} \pm 1{ }^{\circ} \mathrm{C}$ & 6.4 & Good & $\begin{array}{l}\text { No change } \\
\text { in colour }\end{array}$ & Good & Emollient & $\begin{array}{c}\text { Non } \\
\text { greasy }\end{array}$ & Easy \\
\hline & \multirow[t]{2}{*}{5} & RT & 6.7 & Good & $\begin{array}{l}\text { No change } \\
\text { in colour }\end{array}$ & Good & Emollient & $\begin{array}{l}\text { Non } \\
\text { greasy }\end{array}$ & Easy \\
\hline & & $40^{\circ} \mathrm{C} \pm 1{ }^{\circ} \mathrm{C}$ & 6.6 & Good & $\begin{array}{l}\text { No change } \\
\text { in colour }\end{array}$ & Good & Emollient & $\begin{array}{l}\text { Non } \\
\text { greasy }\end{array}$ & Easy \\
\hline & \multirow[t]{2}{*}{10} & RT & 6.8 & Good & $\begin{array}{l}\text { No change } \\
\text { in colour }\end{array}$ & Good & Emollient & $\begin{array}{c}\text { Non } \\
\text { greasy }\end{array}$ & Easy \\
\hline & & $40^{\circ} \mathrm{C} \pm 1{ }^{\circ} \mathrm{C}$ & 6.7 & Satisfactory & $\begin{array}{l}\text { No change } \\
\text { in colour }\end{array}$ & Good & Emollient & $\begin{array}{l}\text { Non } \\
\text { greasy }\end{array}$ & Easy \\
\hline & \multirow[t]{2}{*}{15} & RT & 6.8 & Good & $\begin{array}{l}\text { No change } \\
\text { in colour }\end{array}$ & Good & Emollient & $\begin{array}{c}\text { Non } \\
\text { greasy }\end{array}$ & Easy \\
\hline & & $40^{\circ} \mathrm{C} \pm 1{ }^{\circ} \mathrm{C}$ & 6.8 & Satisfactory & $\begin{array}{l}\text { No change } \\
\text { in colour }\end{array}$ & Poor & Emollient & $\begin{array}{l}\text { Non } \\
\text { greasy }\end{array}$ & Easy \\
\hline & \multirow[t]{2}{*}{20} & RT & 6.9 & Good & $\begin{array}{l}\text { No change } \\
\text { in colour }\end{array}$ & Poor & Emollient & $\begin{array}{l}\text { Non } \\
\text { greasy }\end{array}$ & Easy \\
\hline & & $40^{\circ} \mathrm{C} \pm 1{ }^{\circ} \mathrm{C}$ & 6.8 & Satisfactory & $\begin{array}{l}\text { No change } \\
\text { in colour }\end{array}$ & Poor & Emollient & $\begin{array}{c}\text { Non } \\
\text { greasy }\end{array}$ & Easy \\
\hline
\end{tabular}

Table 5:e: Physical parameter of F5 cream on room and accelerated temperature

\begin{tabular}{|c|c|c|c|c|c|c|c|c|c|}
\hline $\begin{array}{c}\text { Formulatio } \\
\mathbf{n}\end{array}$ & $\begin{array}{c}\text { Day } \\
\text { s }\end{array}$ & $\begin{array}{c}\text { Temperatu } \\
\text { re }\end{array}$ & pH & Homogenity & Appearance & $\begin{array}{c}\text { Spreadibilit } \\
\mathbf{y}\end{array}$ & After feel & $\begin{array}{c}\text { Type } \\
\text { of } \\
\text { smear }\end{array}$ & Removal \\
\hline \multirow{10}{*}{ F5 } & \multirow[t]{2}{*}{0} & RT & 6.7 & Good & $\begin{array}{l}\text { No change } \\
\text { in colour }\end{array}$ & Good & Emollient & $\begin{array}{l}\text { Non } \\
\text { greasy }\end{array}$ & Easy \\
\hline & & $\begin{array}{c}40^{\circ} \mathrm{C} \pm 1 \\
{ }^{\circ} \mathrm{C}\end{array}$ & 6.5 & Good & $\begin{array}{l}\text { No change } \\
\text { in colour }\end{array}$ & Good & Emollient & $\begin{array}{l}\text { Non } \\
\text { greasy }\end{array}$ & Easy \\
\hline & \multirow[t]{2}{*}{5} & RT & 6.8 & Good & $\begin{array}{l}\text { No change } \\
\text { in colour }\end{array}$ & Good & Emollient & $\begin{array}{l}\text { Non } \\
\text { greasy }\end{array}$ & Easy \\
\hline & & $\begin{array}{c}40{ }^{\circ} \mathrm{C} \pm 1 \\
{ }^{\circ} \mathrm{C} \\
\end{array}$ & 6.6 & Satisfactory & $\begin{array}{l}\text { No change } \\
\text { in colour }\end{array}$ & Good & Emollient & $\begin{array}{c}\text { Non } \\
\text { greasy }\end{array}$ & Easy \\
\hline & \multirow[t]{2}{*}{10} & RT & 6.9 & Good & $\begin{array}{l}\text { No change } \\
\text { in colour }\end{array}$ & Low & Emollient & $\begin{array}{l}\text { Non } \\
\text { greasy }\end{array}$ & Easy \\
\hline & & $\begin{array}{c}40^{\circ} \mathrm{C} \pm 1 \\
{ }^{\circ} \mathrm{C}\end{array}$ & 6.7 & Satisfactory & $\begin{array}{l}\text { No change } \\
\text { in colour }\end{array}$ & Low & Emollient & $\begin{array}{l}\text { Non } \\
\text { greasy }\end{array}$ & Easy \\
\hline & \multirow[t]{2}{*}{15} & RT & 7.1 & Good & $\begin{array}{l}\text { No change } \\
\text { in colour }\end{array}$ & Low & Emollient & $\begin{array}{c}\text { Non } \\
\text { greasy }\end{array}$ & Easy \\
\hline & & $\begin{array}{c}40{ }^{\circ} \mathrm{C} \pm 1 \\
{ }^{\circ} \mathrm{C}\end{array}$ & 6.8 & Satisfactory & $\begin{array}{l}\text { No change } \\
\text { in colour }\end{array}$ & Low & Emollient & $\begin{array}{l}\text { Non } \\
\text { greasy }\end{array}$ & Easy \\
\hline & \multirow[t]{2}{*}{20} & RT & 7.1 & Good & $\begin{array}{l}\text { No change } \\
\text { in colour }\end{array}$ & Poor & Emollient & $\begin{array}{l}\text { Non } \\
\text { greasy }\end{array}$ & Easy \\
\hline & & $\begin{array}{c}40^{\circ} \mathrm{C} \pm 1 \\
{ }^{\circ} \mathrm{C}\end{array}$ & 6.8 & Satisfactory & $\begin{array}{l}\text { No change } \\
\text { in colour }\end{array}$ & Good & Emollient & $\begin{array}{l}\text { Non } \\
\text { greasy }\end{array}$ & Easy \\
\hline
\end{tabular}

Table 6: Type of Adverse effect of formulations

\begin{tabular}{l|l|l|l|l} 
S.No & Formulation & Irritant & Erythema & Edema
\end{tabular}


Formulation and evaluation of Antiwrinkle activity of Cream and Nano emulsion of Moringa oleifera

\begin{tabular}{|l|l|l|l|l|}
\hline 1. & $\mathbf{F 1}$ & NIL & NIL & NIL \\
\hline 2. & F2 & NIL & NIL & NIL \\
\hline 3. & $\mathbf{F 3}$ & NIL & NIL & NIL \\
\hline 4. & $\mathbf{F 4}$ & NIL & NIL & NIL \\
\hline 5. & $\mathbf{F 5}$ & NIL & NIL & NIL \\
\hline
\end{tabular}

Table 7: Test for acid value and saponification value

\begin{tabular}{|c|l|c|c|}
\hline S.No & Formulation & Acid value & Saponification value \\
\hline 1. & F1 & 6.7 & 29.2 \\
\hline 2. & F2 & 6.4 & 27.5 \\
\hline 3. & F3 & 5.7 & 25.6 \\
\hline 4. & F4 & 5.5 & 22.1 \\
\hline 5. & F5 & 6.4 & 21.3 \\
\hline
\end{tabular}

Table 8: Rheological study

\begin{tabular}{|c|c|c|c|}
\hline \multirow{2}{*}{ Formulations } & \multirow{2}{*}{ Days } & \multicolumn{2}{|c|}{ Viscosity (cps) } \\
\cline { 3 - 4 } & & Room temperature & $\mathbf{4 5}^{\circ} \mathbf{C}$ \\
\hline 1. & $\mathbf{0}$ & 27500 & 27000 \\
\hline 2. & $\mathbf{5}$ & 28500 & 26000 \\
\hline 3. & $\mathbf{1 0}$ & 30000 & 24000 \\
\hline 4. & $\mathbf{1 5}$ & 31000 & 22500 \\
\hline 5. & $\mathbf{2 0}$ & 32000 & 21000 \\
\hline
\end{tabular}

Table 9: Moringa oleifera nanoemulsion Mean particle diameters (nm) and (PDI)

\begin{tabular}{|c|l|c|c|}
\hline S.No & $\begin{array}{l}\text { Formulation } \\
\text { code }\end{array}$ & $\begin{array}{c}\text { Mean particle } \\
\text { diameters (nm) }\end{array}$ & $\begin{array}{c}\text { Polydispersity } \\
\text { Index (PDI) }\end{array}$ \\
\hline 1. & MONE 1 & $67 \pm 5$ & $0.36 \pm 0.03$ \\
\hline 2. & MONE 2 & $57 \pm 4$ & $0.28 \pm 0.01$ \\
\hline 3. & MONE 3 & $51 \pm 1$ & $0.22 \pm 0.04$ \\
\hline 4. & MONE 4 & $53 \pm 5$ & $0.37 \pm 0.06$ \\
\hline 5. & MONE 5 & $62 \pm 2$ & $0.24 \pm 0.05$ \\
\hline
\end{tabular}

Table 10: Moringa oleifera nanoemulsions zeta potential $(\mathrm{mV})$

\begin{tabular}{|c|l|c|}
\hline S.No & $\begin{array}{l}\text { Formulation } \\
\text { code }\end{array}$ & zeta potential $(\mathbf{m V})$ \\
\hline 1. & MONE 1 & $29 \pm 2.1$ \\
\hline 2. & MONE 2 & $31 \pm 4.2$ \\
\hline 3. & MONE 3 & $43 \pm 1.5$ \\
\hline 4. & MONE 4 & $39 \pm 2.5$ \\
\hline 5. & MONE 5 & $35 \pm 3.4$ \\
\hline
\end{tabular}

Table 11-a: Analysis of the visual evaluation of the reduction in number of wrinkles in $\mathbf{3} \mathrm{cm}$ square area after applying M.olifera oil nanoemulsion formulation.

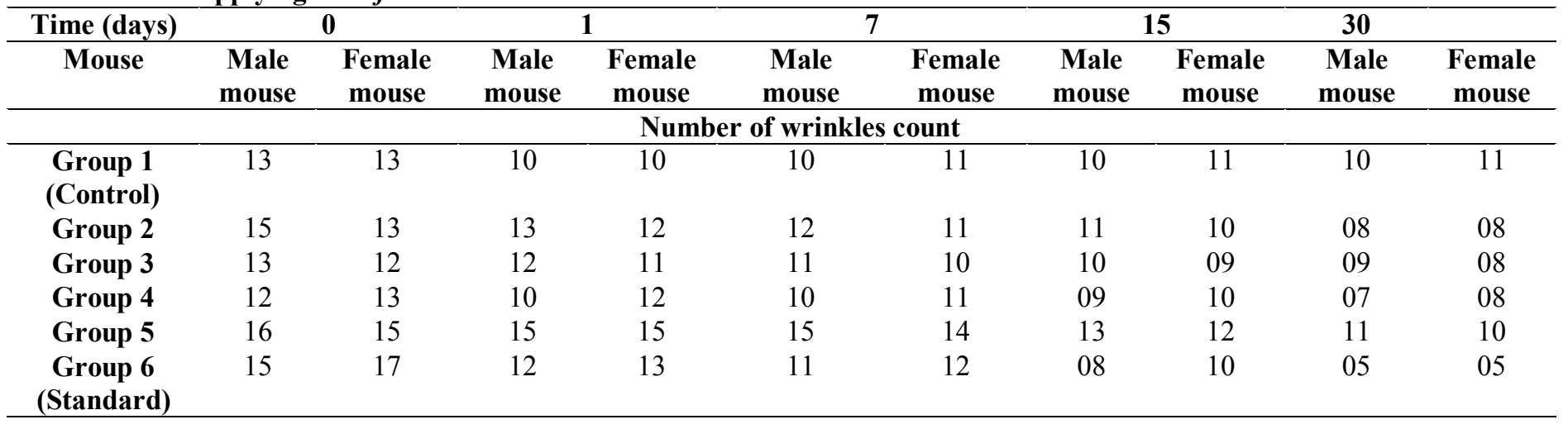


Table 11-b: Analysis of the visual evaluation of the reduction in number of wrinkles in $1 \mathrm{~cm}$ square area after applying M.olifera oil cream formulation.

\begin{tabular}{|c|c|c|c|c|c|c|c|c|c|c|}
\hline \multirow{2}{*}{$\begin{array}{c}\text { Time (days) } \\
\text { Mouse }\end{array}$} & \multicolumn{2}{|c|}{$\mathbf{0}$} & \multicolumn{2}{|c|}{1} & \multicolumn{2}{|c|}{7} & \multicolumn{2}{|c|}{15} & \multicolumn{2}{|l|}{30} \\
\hline & $\begin{array}{l}\text { Male } \\
\text { mouse }\end{array}$ & $\begin{array}{c}\text { Female } \\
\text { mouse }\end{array}$ & $\begin{array}{l}\text { Male } \\
\text { mouse }\end{array}$ & $\begin{array}{c}\text { Female } \\
\text { mouse }\end{array}$ & $\begin{array}{l}\text { Male } \\
\text { mouse }\end{array}$ & $\begin{array}{c}\text { Female } \\
\text { mouse }\end{array}$ & $\begin{array}{l}\text { Male } \\
\text { mouse }\end{array}$ & $\begin{array}{c}\text { Female } \\
\text { mouse }\end{array}$ & $\begin{array}{l}\text { Male } \\
\text { mouse }\end{array}$ & $\begin{array}{c}\text { Female } \\
\text { mouse }\end{array}$ \\
\hline \multicolumn{11}{|c|}{ Number of wrinkles count } \\
\hline Group 2 & 13 & 12 & 13 & 12 & 12 & 11 & 11 & 10 & 09 & 09 \\
\hline Group 3 & 12 & 12 & 12 & 11 & 11 & 10 & 10 & 09 & 09 & 08 \\
\hline Group 5 & 15 & 16 & 15 & 15 & 15 & 14 & 13 & 13 & 12 & 11 \\
\hline $\begin{array}{c}\text { Group } 6 \\
\text { (Standard) }\end{array}$ & 14 & 17 & 12 & 13 & 11 & 12 & 08 & 10 & 05 & 05 \\
\hline
\end{tabular}

Table 12-a: Shows analysis of the visual evaluation of the reduction in size of wrinkles $(\mathrm{mm})$ in $3 \mathrm{~cm}$ square area after applying M.olifera oil nanoemulsion formulation.

\begin{tabular}{|c|c|c|c|c|c|c|c|c|c|c|}
\hline \multirow{2}{*}{$\frac{\text { Time (days) }}{\text { Mouse }}$} & \multicolumn{2}{|r|}{$\mathbf{0}$} & \multicolumn{2}{|c|}{1} & \multicolumn{2}{|c|}{7} & \multicolumn{2}{|c|}{15} & 30 & \multirow[b]{2}{*}{$\begin{array}{c}\text { Female } \\
\text { mouse }\end{array}$} \\
\hline & $\begin{array}{l}\text { Male } \\
\text { mouse }\end{array}$ & $\begin{array}{c}\text { Female } \\
\text { mouse }\end{array}$ & $\begin{array}{l}\text { Male } \\
\text { mouse }\end{array}$ & $\begin{array}{c}\text { Female } \\
\text { mouse }\end{array}$ & $\begin{array}{l}\text { Male } \\
\text { mouse }\end{array}$ & $\begin{array}{c}\text { Female } \\
\text { mouse }\end{array}$ & $\begin{array}{l}\text { Male } \\
\text { mouse }\end{array}$ & $\begin{array}{c}\text { Female } \\
\text { mouse }\end{array}$ & $\begin{array}{l}\text { Male } \\
\text { mouse }\end{array}$ & \\
\hline \multicolumn{11}{|c|}{ Number of wrinkles count } \\
\hline Group 1 & $9.0 \pm$ & $9.0 \pm$ & $9.0 \pm$ & $9.0 \pm$ & $9.0 \pm$ & $9.0 \pm$ & $9.0 \pm$ & $9.10 \pm$ & $9.0 \pm$ & $9.0 \pm$ \\
\hline (Control) & 0.03 & 0.05 & 0.17 & 0.15 & 0.33 & 0.15 & 0.13 & 0.09 & 0.12 & 0.35 \\
\hline \multirow[t]{2}{*}{ Group 2} & $9.0 \pm$ & $9.0 \pm$ & $8.0 \pm$ & $80 \pm$ & $7.0 \pm$ & $7.0 \pm$ & $6.0 \pm$ & $6.10 \pm$ & $5.0 \pm$ & $6.0 \pm$ \\
\hline & 0.07 & 0.11 & 0.09 & 0.10 & 0.12 & 0.14 & 0.16 & 0.15 & 0.17 & 0.12 \\
\hline \multirow[t]{2}{*}{ Group 3} & $13.0 \pm$ & $12.0 \pm$ & $13.0 \pm$ & $120 \pm$ & $11.0 \pm$ & $10.0 \pm$ & $9.0 \pm$ & $9.10 \pm$ & $7.0 \pm$ & $5.0 \pm$ \\
\hline & 0.04 & 0.10 & 0.07 & 0.13 & 0.33 & 0.09 & 0.13 & 0.23 & 0.15 & 0.15 \\
\hline \multirow[t]{2}{*}{ Group 4} & $15.0 \pm$ & $13.0 \pm$ & $14.0 \pm$ & $130 \pm$ & $13.0 \pm$ & $12.0 \pm$ & $12.0 \pm$ & $10.10 \pm$ & $8.0 \pm$ & $8.0 \pm$ \\
\hline & 0.12 & 0.15 & 0.06 & 0.15 & 0.16 & 0.15 & 0.19 & 0.09 & 0.12 & 0.11 \\
\hline \multirow[t]{2}{*}{ Group 5} & $14.0 \pm$ & $12.0 \pm$ & $14.0 \pm$ & $120 \pm$ & $13.0 \pm$ & $11.0 \pm$ & $12.0 \pm$ & $10.10 \pm$ & $11.0 \pm$ & $10.0 \pm$ \\
\hline & 0.15 & 0.22 & 0.10 & 0.11 & 0.54 & 0.08 & 0.09 & 0.09 & 0.19 & 0.35 \\
\hline Group 6 & $10.0 \pm$ & $10.0 \pm$ & $10.0 \pm$ & $100 \pm$ & $9.0 \pm$ & $7.0 \pm$ & $6.0 \pm$ & $6.10 \pm$ & $5.0 \pm$ & $5.0 \pm$ \\
\hline (Standard) & 0.11 & 0.21 & 0.10 & 0.19 & 0.30 & 0.18 & 0.05 & 0.25 & 0.12 & 0.32 \\
\hline
\end{tabular}

Table 12-b: Shows analysis of the visual evaluation of the reduction in size of wrinkles $(\mathrm{mm})$ in $1 \mathrm{~cm}$ square area after applying M.olifera oil cream formulation.

\begin{tabular}{|c|c|c|c|c|c|c|c|c|c|c|}
\hline \multirow{2}{*}{$\begin{array}{c}\text { Time (days) } \\
\text { Mouse }\end{array}$} & \multicolumn{2}{|c|}{$\mathbf{0}$} & \multicolumn{2}{|c|}{1} & \multicolumn{2}{|c|}{7} & \multicolumn{2}{|c|}{15} & \multicolumn{2}{|l|}{30} \\
\hline & $\begin{array}{l}\text { Male } \\
\text { mouse }\end{array}$ & $\begin{array}{c}\text { Female } \\
\text { mouse }\end{array}$ & $\begin{array}{l}\text { Male } \\
\text { mouse }\end{array}$ & $\begin{array}{c}\text { Female } \\
\text { mouse }\end{array}$ & $\begin{array}{c}\text { Male } \\
\text { mouse }\end{array}$ & $\begin{array}{c}\text { Female } \\
\text { mouse }\end{array}$ & $\begin{array}{l}\text { Male } \\
\text { mouse }\end{array}$ & $\begin{array}{c}\text { Female } \\
\text { mouse }\end{array}$ & $\begin{array}{l}\text { Male } \\
\text { mouse }\end{array}$ & $\begin{array}{c}\text { Female } \\
\text { mouse }\end{array}$ \\
\hline \multicolumn{11}{|c|}{ Number of wrinkles count } \\
\hline Group 1 & $8.0 \pm$ & $12.0 \pm$ & $8.0 \pm$ & $12.0 \pm$ & $8.0 \pm$ & $12.0 \pm$ & $8.0 \pm$ & $12.10 \pm$ & $9.0 \pm$ & $14.0 \pm$ \\
\hline (Control) & 0.13 & 0.07 & 0.11 & 0.10 & 0.12 & 0.15 & 0.14 & 0.02 & 0.11 & 0.30 \\
\hline \multirow[t]{2}{*}{ Group 2} & $10.0 \pm$ & $11.0 \pm$ & $8.0 \pm$ & $8.0 \pm$ & $7.0 \pm$ & $7.0 \pm$ & $6.0 \pm$ & $6.10 \pm$ & $5.0 \pm$ & $6.0 \pm$ \\
\hline & 0.11 & 0.08 & 0.09 & 0.15 & 0.33 & 0.10 & 0.16 & 0.09 & 0.12 & 0.12 \\
\hline \multirow[t]{2}{*}{ Group 3} & $12.0 \pm$ & $13.0 \pm$ & $12.0 \pm$ & $12.0 \pm$ & $11.0 \pm$ & $10.0 \pm$ & $9.0 \pm$ & $9.10 \pm$ & $7.0 \pm$ & $7.0 \pm$ \\
\hline & 0.21 & 0.18 & 0.07 & 0.13 & 0.12 & 0.15 & 0.13 & 0.05 & 0.12 & 0.35 \\
\hline \multirow[t]{2}{*}{ Group 4} & $13.0 \pm$ & $12.0 \pm$ & $14.0 \pm$ & $13.0 \pm$ & $13.0 \pm$ & $12.0 \pm$ & $12.0 \pm$ & $10.10 \pm$ & $10.0 \pm$ & $10.0 \pm$ \\
\hline & 0.33 & 0.16 & 0.06 & 0.11 & 0.33 & 0.18 & 0.13 & 0.09 & 0.15 & 0.05 \\
\hline \multirow[t]{2}{*}{ Group 5} & $13.0 \pm$ & $12.0 \pm$ & $13.0 \pm$ & $12.0 \pm$ & $13.0 \pm$ & $11.0 \pm$ & $12.0 \pm$ & $10.10 \pm$ & $11.0 \pm$ & $10.0 \pm$ \\
\hline & 0.11 & 0.11 & 0.07 & 0.15 & 0.54 & 0.15 & 0.19 & 0.10 & 0.12 & 0.35 \\
\hline Group 6 & $11.0 \pm$ & $11.0 \pm$ & $11.0 \pm$ & $10.0 \pm$ & $9.0 \pm$ & $10.0 \pm$ & $6.0 \pm$ & $6.0 \pm$ & $5.0 \pm$ & $5.0 \pm$ \\
\hline (Standard) & 0.09 & 0.43 & 0.15 & 0.10 & 0.33 & 0.16 & 0.10 & 0.09 & 0.4 & 0.09 \\
\hline
\end{tabular}




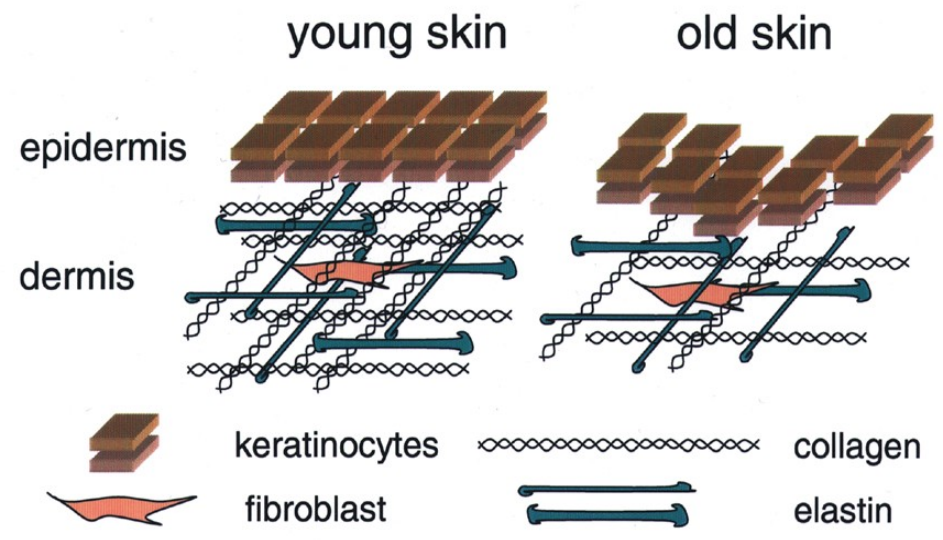

Figure 1: Schematic drawing of young and old skin.

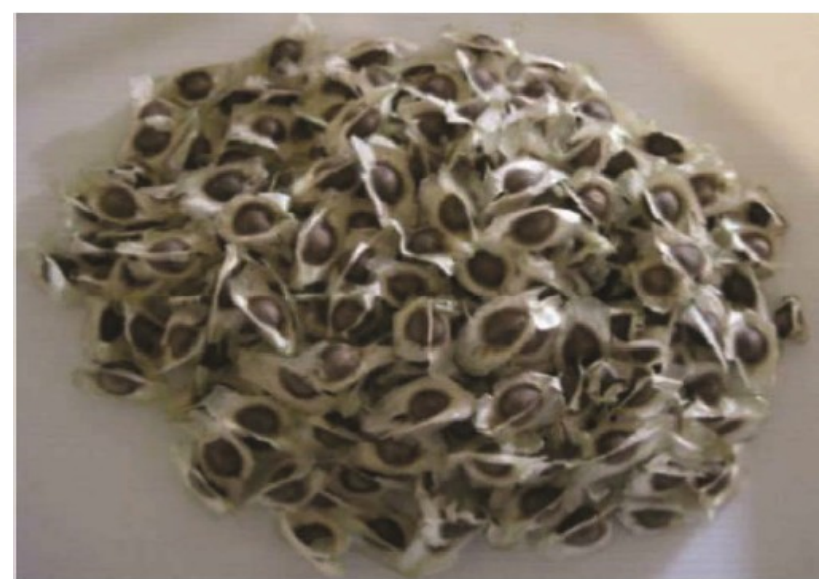

Figure 2: Moringa oleifera seeds as collected from the fruit

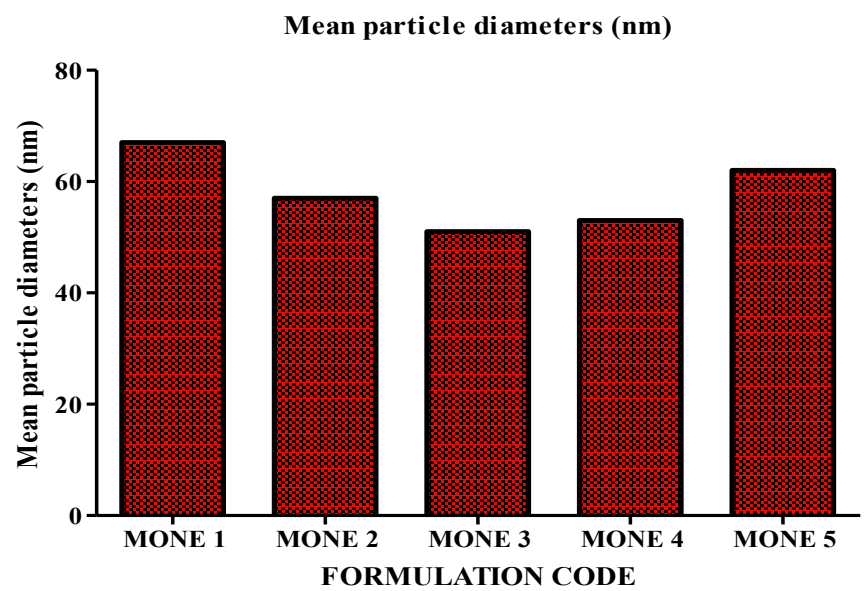

Figure 3: Moringa oleifera nanoemulsions Mean particle diameters (nm) 


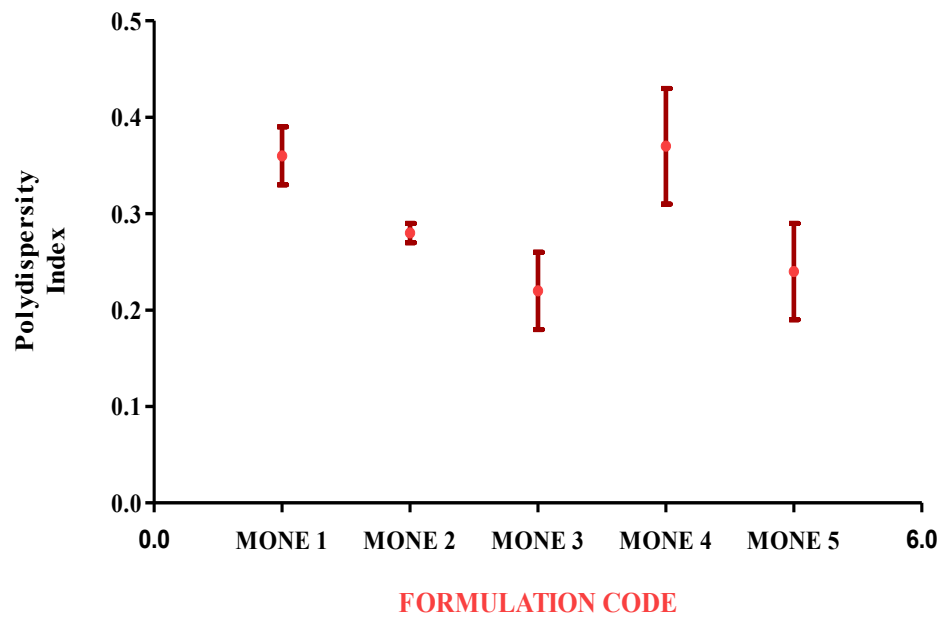

Figure 4: Moringa oleifera nanoemulsions Polydispersity Index (PDI)

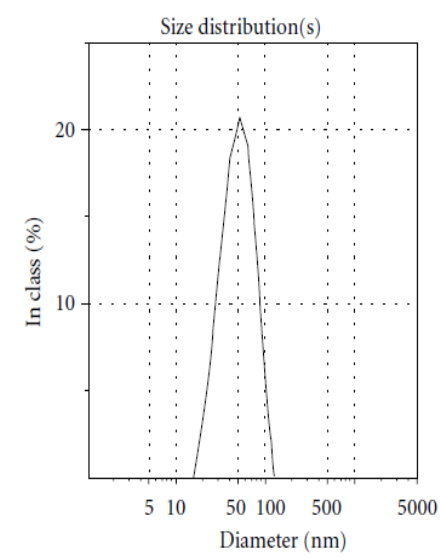

(a)

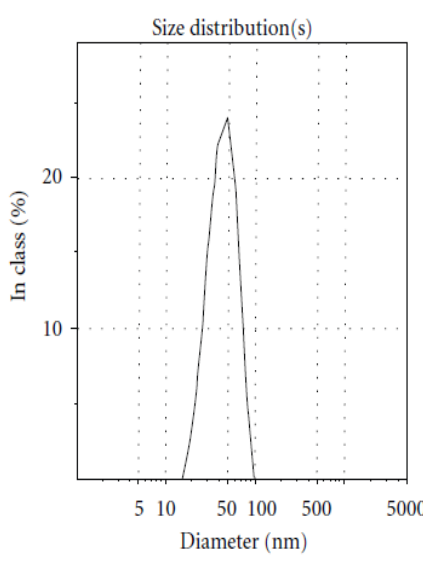

(b)

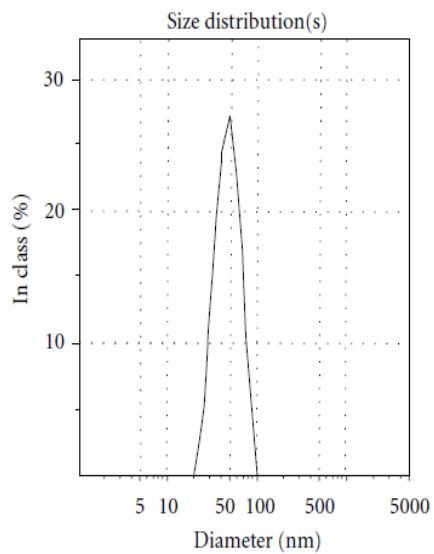

(c)

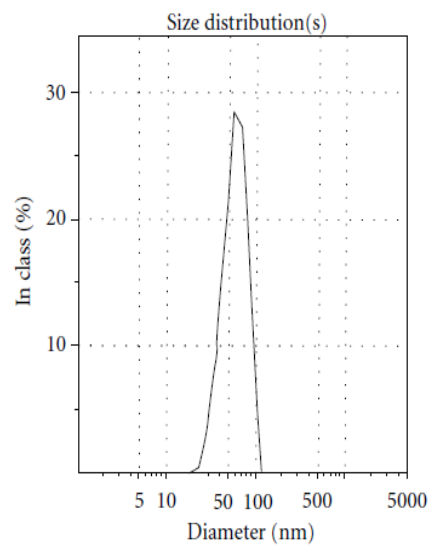

(d)

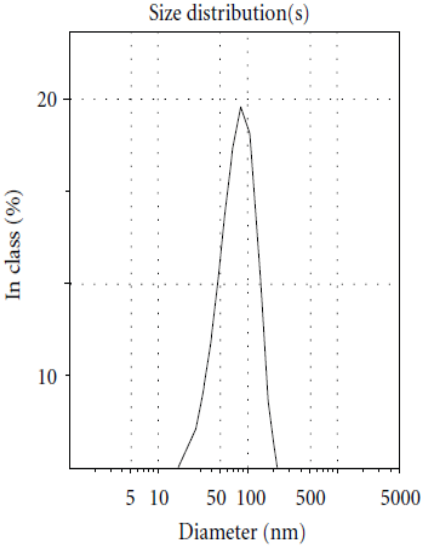

(e)

Figure 5: Moringa oleifera nanoemulsions mean particle diameters (nm), a: MONE 1 b: MONE 2, c: MONE 3, d: MONE 4, e: MONE 5. 


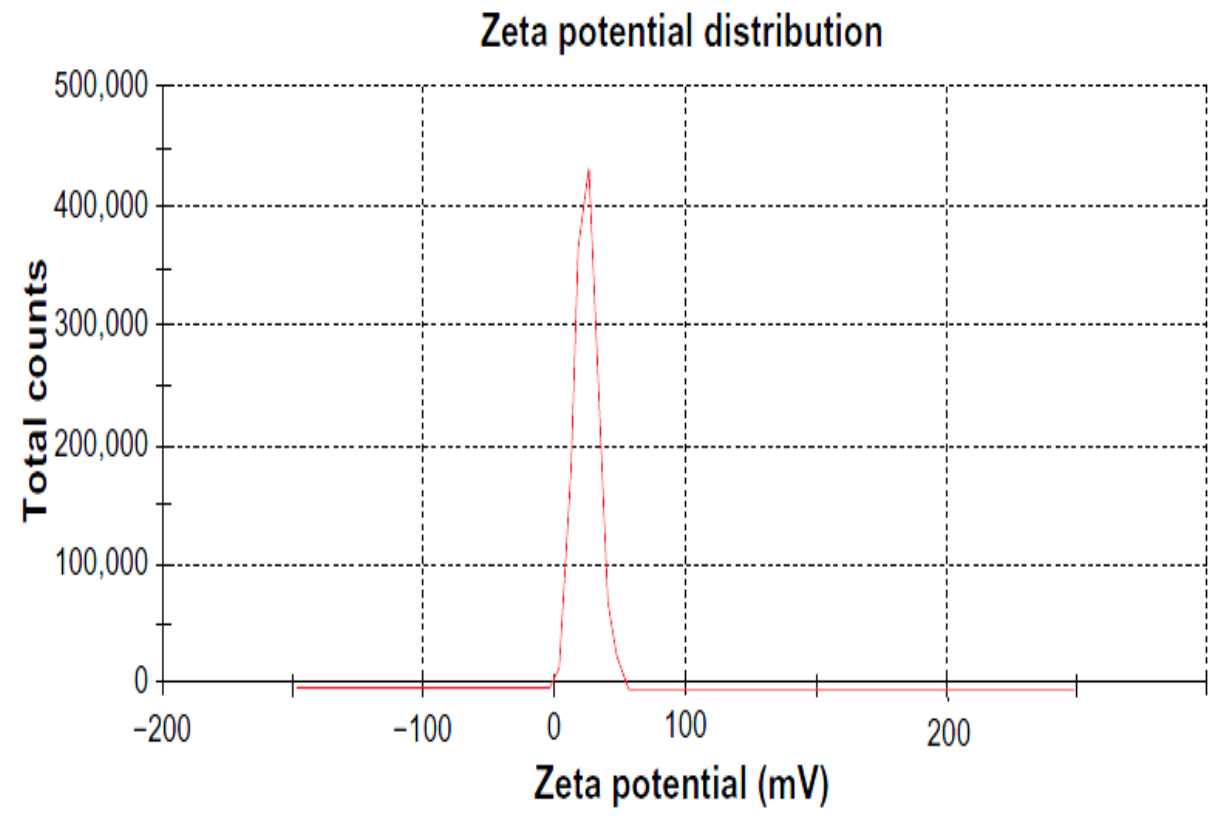

Figure 6: MONE 3 zeta potential $(\mathrm{mV})$

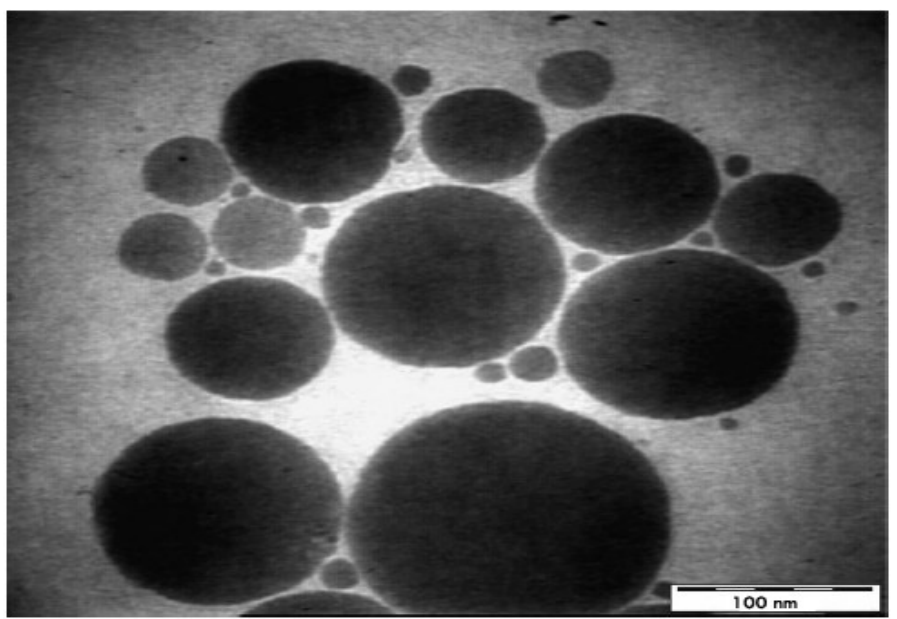

Figure 7: TEM photographs of the MONE 3 


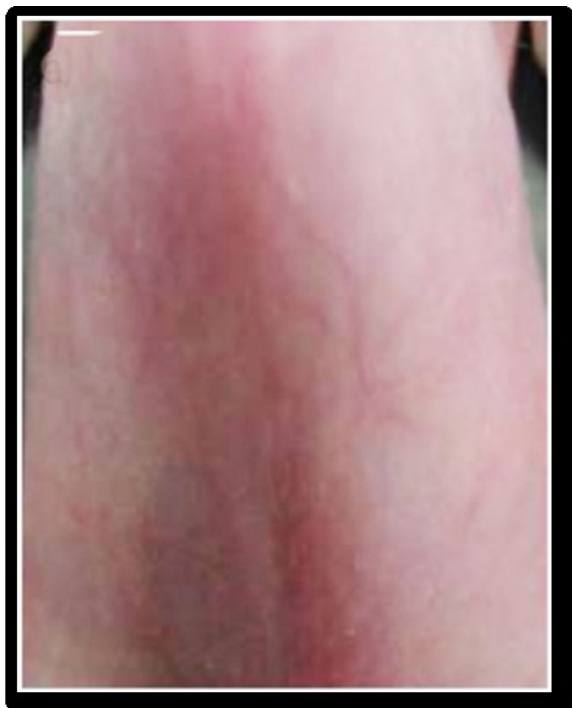

Group 1: normal skin

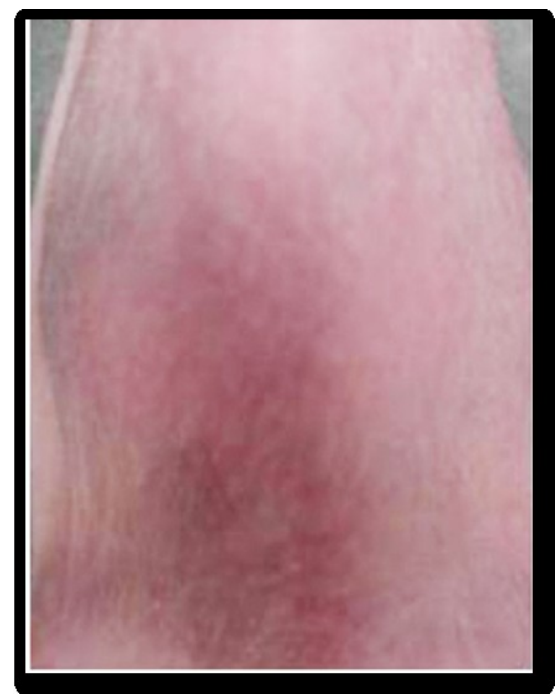

Group 3: showed very smooth skin surface

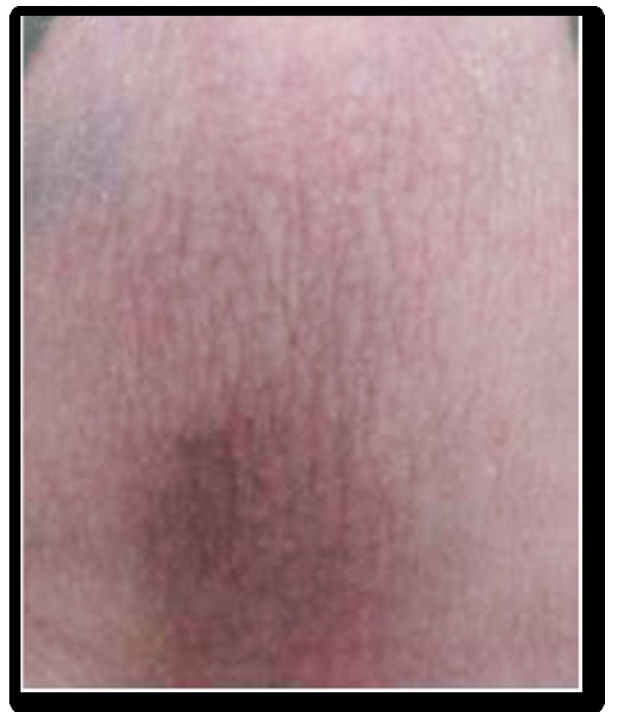

Group 2: Thick and deep wrinkles were observed

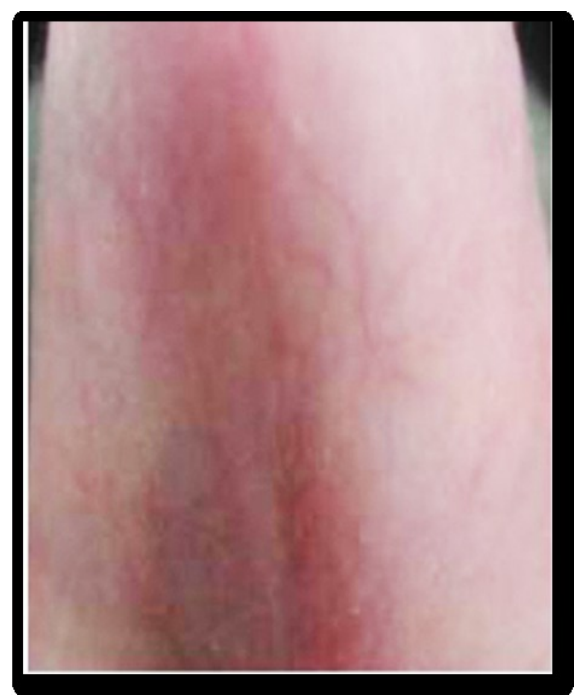

Group 4: showed very smooth skin surface

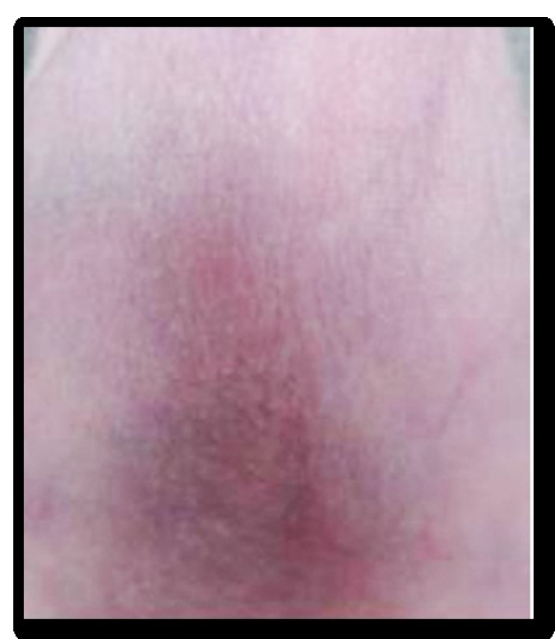

Group 5: showed very smooth skin surface

Figure 8: Anti - wrinkle score of experimental animals 


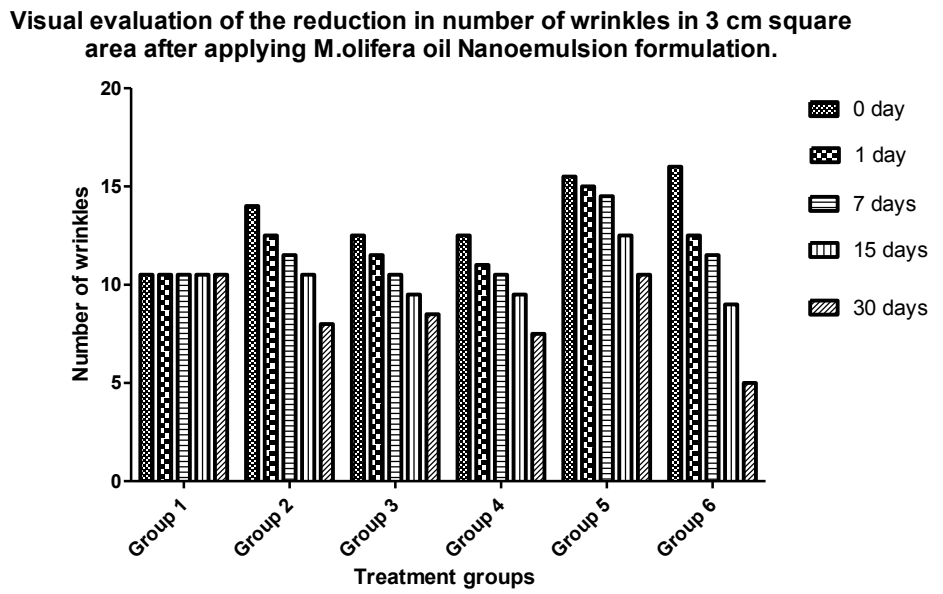

Figure 9-a: Shows analysis of the visual evaluation of the reduction in number of wrinkles in $\mathbf{3} \mathrm{cm}$ square area after applying M.olifera oil nanoemulsion formulation.

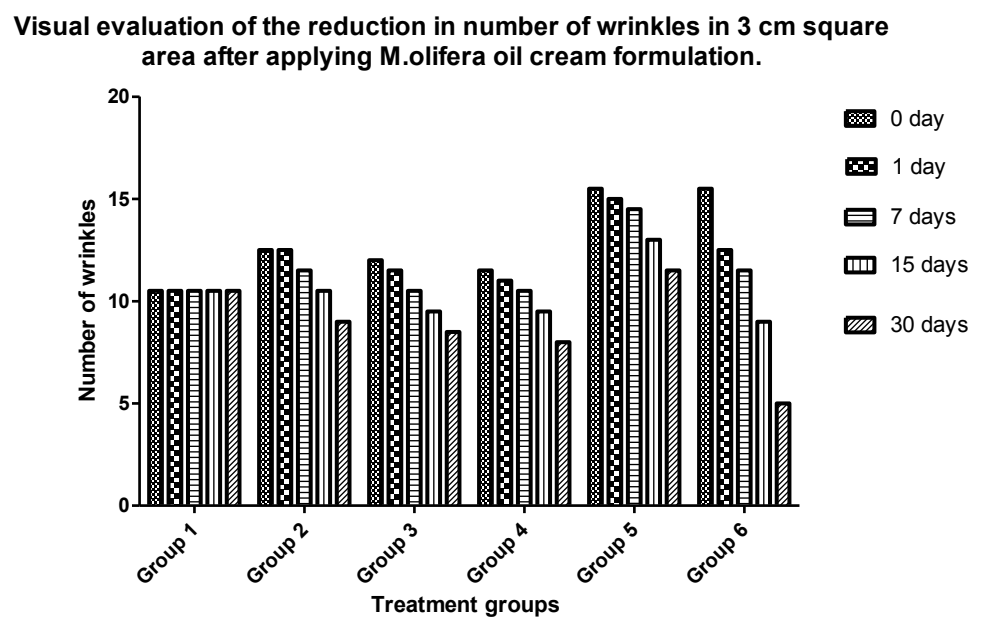

Figure 9-b: Shows analysis of the visual evaluation of the reduction in number of wrinkles in $\mathbf{3} \mathrm{cm}$ square area after applying M.olifera oil cream formulation.

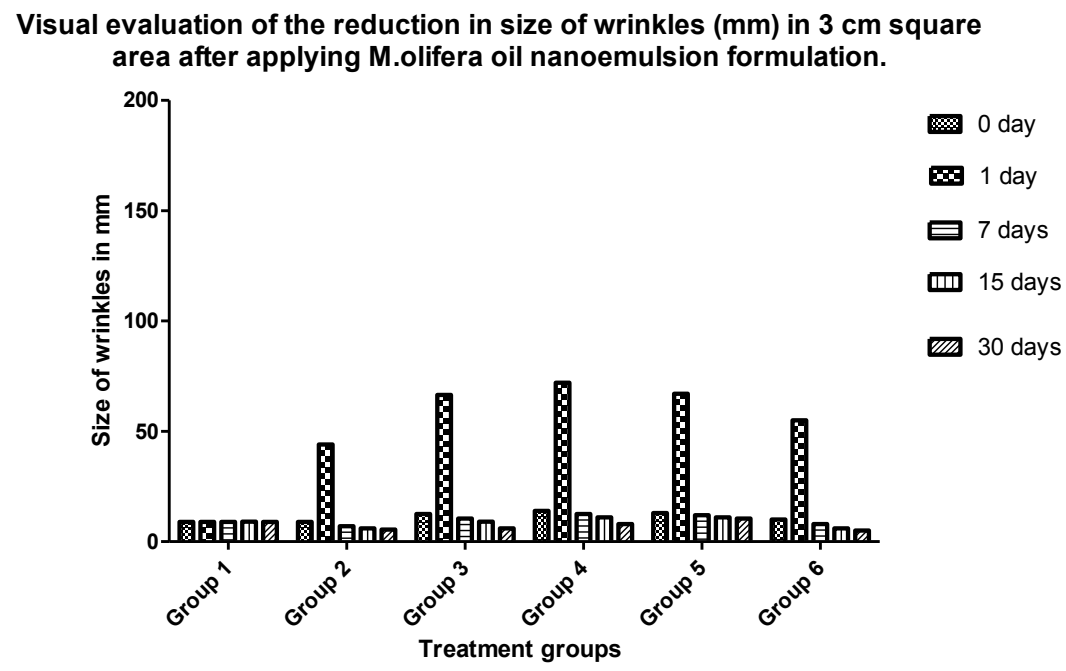

Figure 10-a: Shows analysis of the visual evaluation of the reduction in size of wrinkles $(\mathrm{mm})$ in $3 \mathrm{~cm}$ square area after applying M.olifera oil nanoemulsion formulation. 


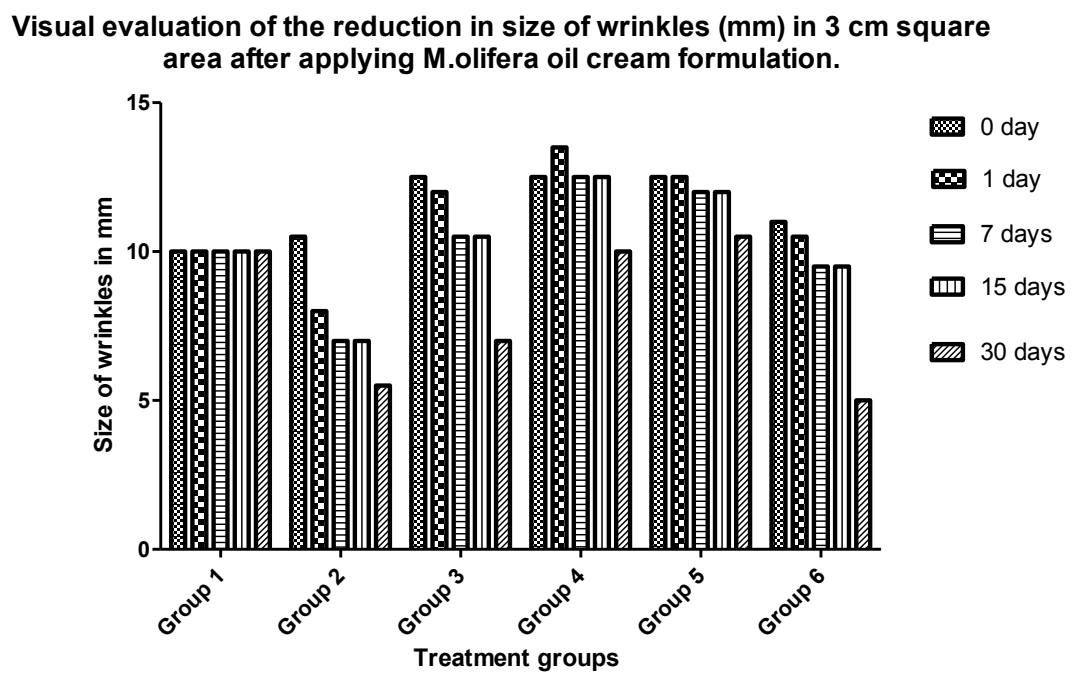

Figure 10-b: Shows analysis of the visual evaluation of the reduction in size of wrinkles $(\mathrm{mm})$ in $3 \mathrm{~cm}$ square area after applying M.olifera oil cream formulation.

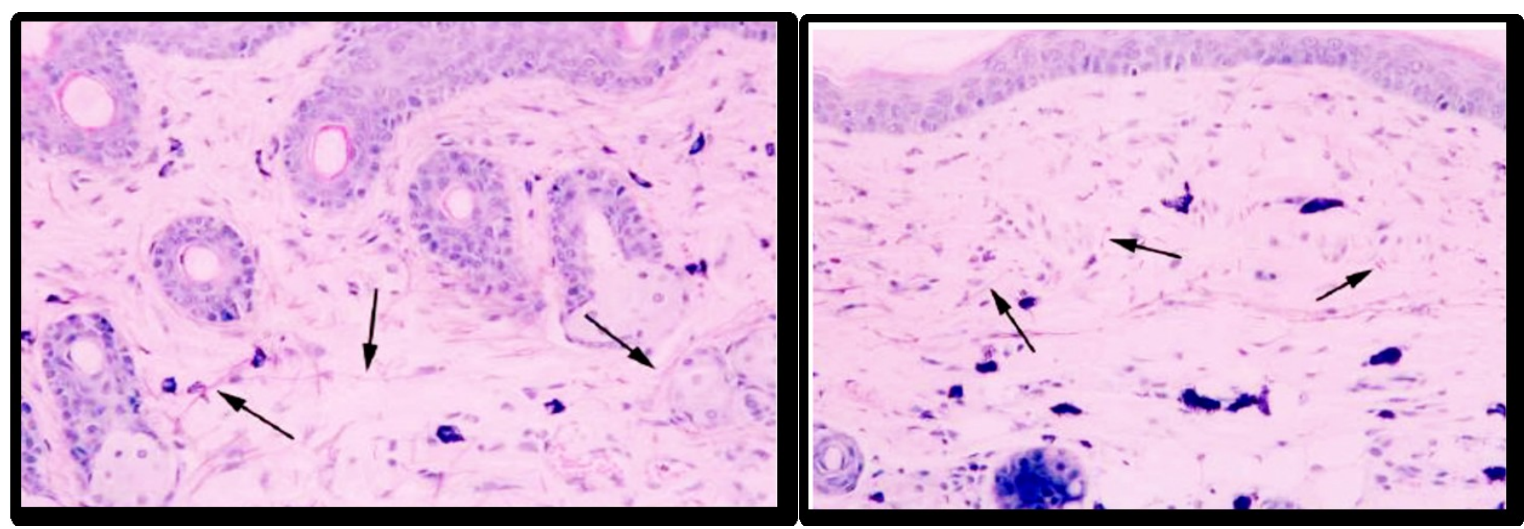

Group 1: normal group

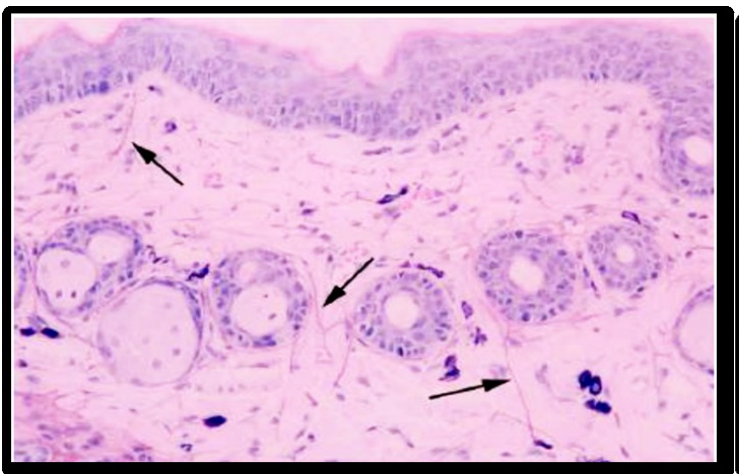

Group: 3
Group 2: The elastic fiber was definitely decreased by UV irradiation

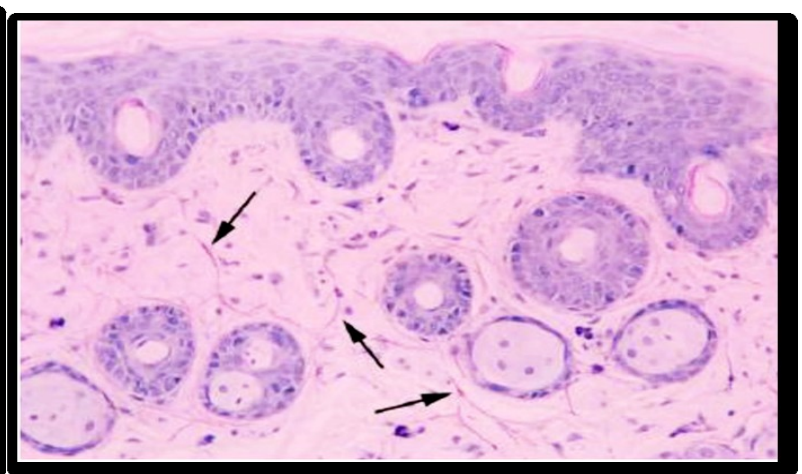

Group 4: The elastic fiber was definitely decreased 


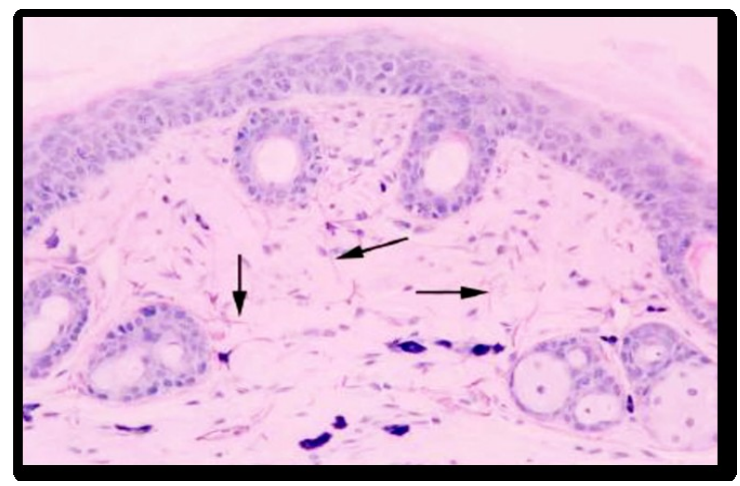

Group: 5

Figure 11: Histological evaluation of mouse skins

\section{Summary and conclusion:}

In the present study, we prepared Moringa oleifera oil based cream and nano-emulsion and system. The prepared cream and nano-emulsion system were characterized and anti-wrinkle effect in vivo was evaluated.

The soxhlet extraction yielded $32.11 \%$ oil extract which is higher when compared ot manual extraction. The Moringa oleifera Oil in water $(\mathrm{O} / \mathrm{W})$ emulsion-based cream was formulated using various concentration of emulsifier. The formulated cream was evaluated for various in vitro physical evaluation tests in room and accelerated temperature upto 20 days and tested at various intervals. The formulation 3 showed $\mathrm{pH}$ 6.0-6.3 in room temperature and $\mathrm{pH} 5.8-6.0$ at $40^{\circ} \mathrm{C}$. The formulated cream showed good homogeneity, Spreadibility, No change in colour and was Emollient, Non greasy and easy to remove. The Moringa oleifera seed oil based cream showed no redness, edema, inflammation and irritation during irritancy studies. The formulation $\mathrm{F} 3$ was safe to use for skin and selected for further in vivo study.

Moringa oleifera nano-emulsion(MONE) was prepared using various concentrations of moringa oil and all the formulations were characterized. All formulations were analyzed directly after production the critical parameters such as particle size, PDI and Zeta potentials were determined. The mean particle diameter of MONE 3 was found to be $51.0 \mathrm{~nm}$. The poly dispersity index of optimized formulation MONE 3 was 0.22 and the Zeta potential $43 \mathrm{mV}$. The MONE 3 nano-emulsion droplets were showed discrete, uniform and spherical with a smooth surface.

The hairless mouse (SKH-1) was used for the in vivo anti-wrinkle efficiency study. The skin wrinkling was induced by UVB irradiation at $365 \mathrm{~nm}$. The anti-wrinkle score was observed visually by taking photographs before treatment. After 30days treatment with the formulations and the standard, the wrinkle score, number of wrinkles and size of wrinkles was observed microscopically and also in photographs and the results were tabulated. The histological examination of skin specimens were stained by the Pinkus' acid orcein-Giemsa staining method. The Moringa oleifera Oil nano-emulsion (MONE 3) showed better protective effect compared to $M$. oleifera Oil o/w cream Formulation-3. The possible mechanism of the anti-wrinkle effect of Moringa oleifera Oil based cream formulations was hypothesized as follows. The nano emulsion delivered to the viable dermal layer where the antioxidant constituents has to be delivered and the delivery was promoted by the hydrating effect on the skin surface. However, due to its hydro phobic nature of $M$. oleifera formulations were deposited mainly into the viable dermal layer. The M. oleifera Oil formulations exerted an anti-oxidative effect, which protected against the loss of elastin, ultimately resulting in the anti-wrinkle effects.

\section{References}

[1]. Mirinda A, Farrage Kenneth, W. Miller and Howard I. Maibach, Text book of aging skin, 2010, ISBN: 978-3-540-89655-5 (Print) 978-3-540-89656-2 (Online), Page No: 25-54.

[2]. L Baumann, Skin Ageing and its treatment, J Pthol 211, 241-51 (2007).

[3]. Gilchrest BA, Skin aging and photoaging: an overview. J Am Acad Dermatol 1989;21:610-3.

[4]. Jin Young Seo, Jin Ho Chung, Thermal aging: A new concept of skin aging, J Dermatol science supplement (2006)2, 513-522.

[5]. Puizina-Ivic N, Miric L, Carija A, Karlica D, Marasovic D, "Modern approach to topical treatment of aging skin", Coll Antropol. 2010 Sep;34(3): 1145-53.

[6]. Atif Ali, Naveed Akhtar, Muhammad Shoaib Khan, Fatima Rasool, Furqan Muhammad Iqbal, Muhammad Tahir Khan, Minhaj Ud Din, Ehsan Elahi, "Moisturizing effect of cream containing Moringa oleifera (Sohajana) leaf extract by biophysical techniques: In vivo evaluation", Journal of Medicinal Plants Research, 2013,Vol. 7(8), pp. 386-391.

[7]. Vikash Kumar, Nishtha Pandey, Nitin Mohan, Ram P. Singh, “Antibacterial \& antioxidant activity of different extract of Moringa oleifera", International Journal of Pharmaceutical Sciences Review and Research, 2012, 12(1): 89-94.

[8]. Genji Imokava, "Mechanism of UVB- induced wrinkling of the skin: paracrine cytokine linkage between keratinocytes and Fibroblasts leading to the stimulation of Elastase", Journal of Investigative Dermatology Symposium Proceedings (2009) 14, 36-43; doi:10,1038/jidsymp.2009. 
[9]. Imokawa G, "Recent advances in characterizing biological mechanisms underlying UV-induced wrinkles: a pivotal role of fibroblast-derived elastase", Arch Dermatol Res. 2008 Apr;300 Suppl 1:S7-20.

[10]. Hye-Sook Han, Youn-Ja Kwon, Myoung-Soon Park, Si-Ho Park, So-Mi Kim Cho, Young-Soy Rho, Jin-Wou Kim, Hong-Sig Sinb and Soo-Jong Uma, "Efficacy Validation of Synthesized Retinol Derivatives - In Vitro: stability, toxicity and activity", Bioorganic \& Medicinal Chemistry, 2003, 11: 3839-3845. 